\title{
A formal language model of DNA Polymerase enzymatic activity
}

\author{
Srujan Kumar Enaganti Lila Kari \\ Steffen Kopecki \\ Department of Computer Science \\ The University of Western Ontario \\ London, ON, Canada
}

\begin{abstract}
We propose and investigate a formal language operation inspired by the naturally occurring phenomenon of DNA primer extension by a DNAtemplate-directed DNA Polymerase enzyme. Given two DNA strings $u$ and $v$, where the shorter string $v$ (called primer) is Watson-Crick complementary and can thus bind to a substring of the longer string $u$ (called template) the result of the primer extension is a DNA string that is complementary to a suffix of the template which starts at the binding position of the primer. The operation of DNA primer extension can be abstracted as a binary operation on two formal languages: a template language $L_{1}$ and a primer language $L_{2}$. We call this language operation $L_{1}$-directed extension of $L_{2}$ and study the closure properties of various language classes, including the classes in the Chomsky hierarchy, under directed extension. Furthermore, we answer the question under what conditions can a given language of target strings be generated from a given template language when the primer language is unknown. We use the canonic inverse of directed extension in order to obtain the optimal solution (the minimal primer language) to this question.
\end{abstract}

\section{Introduction}

Computational models inspired by nature abound in theoretical computer science. Several formal language operations that have their basis on naturally occurring biochemical reactions have been proposed and studied. The actions of various enzymes on DNA strands, most of which are widely used in the field of biotechnology, are of particular interest. In this paper we propose and investigate a formal language operation that models the action of DNA Polymerase enzyme, an enzyme that plays a major role in the replication of DNA strands.

\footnotetext{
${ }^{0}$ This research was supported by a Natural Science and Engineering Council of Canada (NSERC) Discovery Grant and a University of Western Ontario Grant to L.K.
} 
Other bio-inspired operations in the literature include splicing, insertion and deletion, substitution, and hairpin extension. Splicing is a formal language operation originally proposed by Tom Head [10] to model the recombination of DNA strands under the action of restriction enzymes and ligase enzymes. Various types of splicing systems have been developed based on this phenomenon and their properties were studied in, e.g., [29] [9] [19] [11] [15]. Insertion-deletion operations are basic to DNA processing and RNA editing in molecular biology. Insertion-Deletion systems were defined as formal models of computation based on these operations and have been widely studied in the literature, see, e.g., [17] [31] [33] [34] [30] [18] [5]. Insertion-deletion systems that are context-free [27], that have one sided-context [28] [23], and that are graph controlled [6] were also proposed. $P$-systems with insertion-deletion rules have been extensively studied in [22] [24] [2] [1] [7] [8]. A type of substitution operation inspired by errors occurring in biologically encoded information was proposed in [16]. Hairpin formation is a naturally occurring phenomenon whereby a DNA strand that is partially self-complementary attaches to itself. Based on this phenomenon, the formal language operation called hairpin completion as well as its inverse operation called hairpin reduction have been defined and extensively studied in the literature [4] [26] [25] [21].

In this paper we define and investigate a formal language operation that models the action of the DNA Polymerase enzyme on DNA strands. Recall that a DNA single-strand consists of four different types of units called nucleotides or bases strung together by an oriented backbone like beads on a wire. The distinct ends of a DNA single strand are called the 5' end and the 3' end respectively. The bases are Adenine $(A)$, Guanine $(G)$, Cytosine $(C)$ and Thymine $(T)$, and $A$ can chemically bind to an opposing $T$ on another single strand, while $C$ can similarly bind to $G$. Bases that can thus bind are called Watson/Crick $(W / C)$ complementary, and two DNA single strands with opposite orientation and with $W / C$ complementary bases at each position can bind to each other to form a DNA double strand in a process called base-pairing.

The activity of DNA Polymerase presupposes the existence of a DNA single strand called template (Figure 1 (a)), and of a second short DNA strand called primer, that is Watson-Crick complementary to the template (Figure 1 (b)). Given a supply of individual nucleotides, the DNA polymerase enzyme extends the primer, at one of its ends only, by adding invididual nucleotides complementary to the template nucleotides, one by one, until the end of the template is reached (Figure 1 (c)). The newly formed DNA strand is a strand that starts with the primer and is partially Watson-Crick complementary to the template (Figure 1 (d)). In molecular biology laboratories, an iterated version of this process is used to obtain an exponential replication of DNA strands, in a protocol called Polymerase Chain Reaction, or PCR.

In this paper we introduce a simplified formal language model of DNA Polymerase enzymatic activity, called template-directed extension, or simply directed extension. The paper is organized as follows. Section 2 contains definitions and notations, including the definition of directed extension. In Section 3, we give proofs for the closure properties of the various language classes under directed 


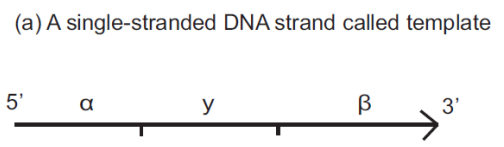

(b) The primer that is complementary to the template

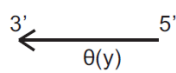

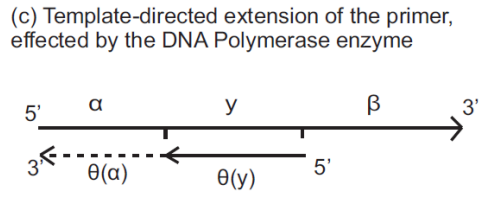

(d) Newly formed output molecule

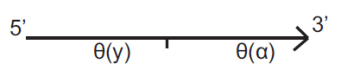

Figure 1: Template directed extension of a primer, effected by DNA Polymerase enzyme. By $\theta(x)$ we denote the Watson-Crick complement of a DNA strand $x$.

extension. In particular, we show that the directed extension between two languages in LOGSPACE can result in an undecidable language. In Section 4, we define an inverse of directed extension and study language equations involving this operation. In Section 5, we compare our operation with related string operations, and we discuss iterated versions of directed extension.

\section{Basic definitions and notations}

An alphabet $\Sigma$ is a finite non-empty set of symbols. $\Sigma^{*}$ denotes the set of all words over $\Sigma$, including the empty word $\lambda . \Sigma^{+}$is the set of all non-empty words over $\Sigma$. For words $w, x, y, z$ such that $w=x y z$ we call the subwords $x$, $y$, and $z$ prefix, infix, and suffix of $z$, respectively. The sets $\operatorname{Pref}(w), \operatorname{Inf}(w)$, and $\operatorname{Suff}(w)$ contain, respectively, all prefixes, infixes, and suffixes of $w$. This notation is extended to languages as follows: $\operatorname{Suff}(L)=\bigcup_{w \in L} \operatorname{Suff}(w)$. The complement of a language $L \subseteq \Sigma^{*}$ is $L^{c}=\Sigma^{*} \backslash L$. By FIN, REG, LIN, CF, CS, and $\mathrm{RE}$ we denote the families of finite, regular, linear (context-free), contextfree, context-sensitive, and recursively enumerable languages, respectively.

An involution is a function $\theta: \Sigma^{*} \rightarrow \Sigma^{*}$ with the property that $\theta^{2}$ is identity. $\theta$ is called an antimorphism if $\theta(u v)=\theta(v) \theta(u)$. Traditionally, the Watson-Crick complementarity of languages has been modelled as an antimorphic involution over the DNA alphabet $\Delta=\{A, C, G, T\},[12,14]$. Assuming the convention that a word $x$ over this alphabet represents the DNA single strand $x$ in the 5 , to 3' direction, the activitity of DNA polymerase in Figure 1, given a template $\alpha y \beta$ and a primer $y$ that occurs only once in $\alpha y \beta$, can be modelled as:

$$
\alpha y \beta \bullet \theta(y)=\theta(y) \theta(\alpha)=\theta(\alpha y) .
$$

Assuming that all involved DNA strands are initially double-stranded, that is, whenever the strand $x$ is available also its Watson-Crick complement $\theta(x)$ is available, we can further simplify this model and, given two words $x, y$ over an alphabet $\Sigma$, we can define the left $x$-directed extension of $y$ as 


$$
x \oplus^{\prime} y=\left\{w \in \Sigma^{*} \mid \exists \alpha, \beta \in \Sigma^{*}: x=\alpha y \beta, w=\alpha y\right\},
$$

and the right $x$-directed extension of $y$ as

$$
x \oplus y=\left\{w \in \Sigma^{*} \mid \exists \alpha, \beta \in \Sigma^{*}: x=\alpha y \beta, w=y \beta\right\},
$$

From a mathematical point of view the left- and right-directed extensions are similar. For the remainder of this paper we will consider only the right-directed extension, which we will call simply directed extension.

Note also that, from a biological point of view, it does not make sense to consider an "empty primer" (a primer with length 0 ), but from a mathematical point of view this is well-defined and $y=\lambda$ is valid. We extend the definition of directed extension to languages in a natural way:

$L_{x} \oplus L_{y}=\bigcup_{x \in L_{x}, y \in L_{y}} x \oplus y=\left\{w \in \Sigma^{+} \mid \exists \alpha, \beta \in \Sigma^{*}, y \in L_{y}: \alpha y \beta \in L_{x}, w=y \beta\right\}$

\section{Closure Properties}

In this section we study closure properties of various language classes under directed extension. Throughout this section all languages are considered to be defined over a fixed alphabet $\Sigma$. The next lemma expresses the directed extension operation in terms of concatenation, intersection and suffix.

Lemma 3.1. If $L_{x}$ and $L_{y}$ are two languages over $\Sigma$, then $L_{x} \oplus L_{y}=\operatorname{Suff}\left(L_{x}\right) \cap$ $L_{y} \Sigma^{*}$.

Proof. For the direct inclusion, consider $w \in L_{x} \oplus L_{y}$. This implies that $w=y \beta$ where $y \in L_{y}$ and $\alpha y \beta \in L_{x}$. Therefore, $w \in L_{y} \Sigma^{*}$ and $w \in \operatorname{Suff}\left(L_{x}\right)$.

Conversely, let $w \in \operatorname{Suff}\left(L_{x}\right) \cap L_{y} \Sigma^{*}$. Because $w \in \operatorname{Suff}\left(L_{x}\right)$, there exists $\alpha \in \Sigma^{*}$ such that $\alpha w \in L_{x}$. Because $w \in L_{y} \Sigma^{*}$, there exists $y \in L_{y}$ and $\beta \in \Sigma^{*}$ such that $w=y \beta$. Thus, $w \in L_{x} \oplus L_{y}$.

Corollary 3.2. Let $\mathcal{X}$ and $\mathcal{Y}$ be two language classes where $\mathcal{X}$ is closed under the suffix operator and $\mathcal{Y}$ is closed under concatenation with $\Sigma^{*}$.

i.) If $\mathcal{X}$ is closed under intersection with languages from $\mathcal{Y}$, then for all $L_{x} \in$ $\mathcal{X}$ and $L_{y} \in \mathcal{Y}$ we have $L_{x} \oplus L_{y} \in \mathcal{X}$.

ii.) If $\mathcal{Y}$ is closed under intersection with languages from $\mathcal{X}$, then for all $L_{x} \in$ $\mathcal{X}$ and $L_{y} \in \mathcal{Y}$ we have $L_{x} \oplus L_{y} \in \mathcal{Y}$.

In particular, REG and $R E$ are closed under directed extension and, if $\mathcal{X}$ is $L I N(C F)$ and $\mathcal{Y}$ is $R E G$, then the result $L_{x} \oplus L_{y}$ is in $L I N(C F)$.

Next, we show that directed extension can "simulate" intersection by utilizing markers at the beginning and end of words. 
Lemma 3.3. Let $L_{1}$ and $L_{2}$ be languages over the alphabet $\Sigma$ and let $\$ \notin \Sigma$ be a new symbol. Then,

$$
\$ L_{1} \$ \oplus \$ L_{2} \$=\$\left(L_{1} \cap L_{2}\right) \$ .
$$

Proof. For the direct inclusion, let $x \in L_{1}$ and $y \in L_{2}$. If the word $\$ x \$$ has a factorization $\$ x \$=\alpha \$ y \$ \beta$, it is clear that $x=y$ and $\alpha=\beta=\lambda$ because $\$$ does not occur as letter in $x$. Therefore, if $w \in \$ x \$ \oplus \$ y \$$ for some $x \in L_{1}$ and $y \in L_{y}$, then $w \in \$\left(L_{1} \cap L_{2}\right) \$$.

For the converse inclusion, let $w$ be any string in $\left(L_{1} \cap L_{2}\right)$. This implies that $\$ w \$ \in \$ L_{1} \$$ and $\$ w \$ \in \$ L_{2} \$$. Thus $\$ w \$ \in \$ L_{1} \$ \oplus \$ L_{2} \$$.

Lemma 3.3 allows us to classify the result of directed extension between two (linear) context-free languages.

Theorem 3.4. Let $L_{x}$ be a context-free language and $L_{y}$ be a context-free (or context-sensitive) language. The language $L_{x} \oplus L_{y}$ is context-sensitive, but not necessarily context-free.

Proof. Consider the two (linear) context-free languages

$$
L_{x}=\left\{\$ a^{m} b^{n} c^{n} \$ \mid m \geq 1, n \geq 1\right\}, \quad L_{y}=\left\{\$ a^{n} b^{n} c^{m} \$ \mid m \geq 1, n \geq 1\right\} .
$$

By Lemma 3.3, the $L_{x}$-directed extension of $L_{y}$ yields the context-sensitive but not context-free language

$$
L_{x} \oplus L_{y}=\left\{\$ a^{n} b^{n} c^{n} \$ \mid n \geq 1\right\} .
$$

In order to show that $L_{x} \oplus L_{y}$ is context-sensitive for $L_{x} \in \mathrm{CF}$ and $L_{y} \in \mathrm{CS}$, we use Lemma 3.1 and note that the suffix operator applied to a context-free language gives a context-free language and that the class of context-sensitive languages is closed under intersection.

Let $L O G=D S P A C E(\log )$ be the language class which contains all languages that can be accepted by a deterministic Turing Machine using at most $\mathcal{O}(\log n)$ space on an input of length $n$. For a language $L_{x} \in L O G$ we will show that the $L_{x}$-directed extension of a singleton language can produce an undecidable language. In order to do so, we utilize the undecidable Post Correspondence Problem (PCP) in the following formulation: Determine, for an arbitrary set $\left(x_{1}, y_{1}\right),\left(x_{2}, y_{2}\right), \cdots,\left(x_{k}, y_{k}\right)$ of pairs of corresponding non-null strings over the alphabet $\{a, b\}$, whether or not there exists a solution $n, i_{1}, i_{2}, i_{3}, \cdots, i_{n}$ such that $x_{i_{1}} x_{i_{2}} x_{i_{3}} \cdots x_{i_{n}}=y_{i_{1}} y_{i_{2}} y_{i_{3}} \cdots y_{i_{n}}, n \geq 1, i_{j} \in\{1,2, \cdots, k\}$.

Theorem 3.5. There exists a language $L_{1}$ in $L O G$ and a singleton language $L_{2}$ such that $L_{1} \oplus L_{2}$ is not decidable.

Proof. Let $L_{1}$ be a language over $\Sigma \cup\{\$\}$ consisting of all strings of the form $\alpha \$ \beta$ where $\$$ does not appear within $\alpha$ or $\beta$. Here $\beta$ is the encoding of an instance of the PCP and $\alpha$ is the encoding of a solution of this instance. We let $L_{2}$ be 
the singleton language $\{\$\}$. The resulting language $L_{1} \oplus L_{2}$ contains all strings of the form $\$ \beta$ such that $\alpha \$ \beta \in L_{1}$; therefore, $\$ \beta \in L_{1} \oplus L_{2}$ if and only if $\beta$ is the encoding of an instance of PCP which has a solution. Formally,

$$
\begin{aligned}
L_{1} & =\{\alpha \$ \beta \mid \beta \text { is a PCP instance and } \alpha \text { is a solution to } \beta\} \\
L_{2} & =\{\$\}, \\
L_{1} \oplus L_{2} & =\{\$ \beta \mid \beta \text { is a PCP instance that has a solution }\} .
\end{aligned}
$$

Because PCP is undecidable, it will follow that the language $L_{1} \oplus L_{2}$ is undecidable as well. Let us show next how to encode $\alpha$ and $\beta$ in a word $\alpha \$ \beta \in L_{x}$ and how to decide $L_{x}$ using logarithmic space.

Let $x_{1}, x_{2}, \ldots, x_{k}$ and $y_{1}, y_{2}, \ldots, y_{k}$ be an instance of PCP and let $i_{1}, i_{2}, \ldots i_{n}$ be a solution to this instance. We encode each integer $i_{j}$ using a binary encoding, symbolized as $\left|i_{j}\right|$, which is of length $\left\lceil\log _{2} k\right\rceil$ or less. Let $\alpha \$ \beta$ be encoded as

$$
\left|i_{1}\right| M\left|i_{2}\right| M\left|i_{3}\right| M \ldots\left|i_{n}\right| M \$ M x_{1} M x_{2} M x_{3} \ldots M x_{k} M C M y_{1} M y_{2} M y_{3} \ldots M y_{k} M
$$

where $M$ and $C$ are separating symbols.

In order to decide if an arbitrary string $w$ is in $L_{1}$, the first step is to verify that it is of the format described above and the second step is to verify that the integer sequence $\alpha$ is a solution of $\beta$. In order to decide $L_{1}$ we have to verify whether or not $x_{i_{1}} x_{i_{2}} x_{i_{3}} \cdots x_{i_{n}}$ and $y_{i_{1}} y_{i_{2}} y_{i_{3}} \cdots y_{i_{n}}$ are equal. We can easily see that the first step can be done in logarithmic space and that the second step can (at least) be decided. Thus, the language $L_{1}$ is decidable.

Now, we give a high-level construction of a Turing Machine which uses logarithmic working space with respect to the length of the input and decides whether $\alpha$ is a solution to $\beta$ or not. Instead of generating both strings completely and then comparing them, we generate and compare both strings letter by letter. In order to do so, we only need to store pointers to the input tape on the work tape which can be implemented using only logarithmic space. A more detailed description of this Turing Machine follows.

We may assume the symbol $S$ is written to the left of input and refer to it as the start symbol. The strings $x_{i_{1}} x_{i_{2}} \cdots x_{i_{n}}$ and $y_{i_{1}} y_{i_{2}} \cdots y_{i_{n}}$ are referred to as $x$ and $y$ respectively.

When we say address, we refer to the address on the input tape with respect to $S$, i.e. the number of symbols we have to move to the right starting from $S$ on the input tape. The input tape looks as follows:

$$
S\left|i_{1}\right| M\left|i_{2}\right| M\left|i_{3}\right| M \ldots\left|i_{n}\right| M \$ M x_{1} M x_{2} M x_{3} \ldots M x_{k} M C M y_{1} M y_{2} M y_{3} \ldots M y_{k} M
$$

The computation of the Turing Machine is described by Algorithm 1. We 
use the following variables in the pseudo-code:

$$
\begin{array}{ll}
x_{a d d r} & - \text { The address of current symbol of } x \text { that is being looked into } \\
y_{a d d r} & - \text { The address of current symbol of } y \text { that is being looked into } \\
x_{\text {soln }} & - \text { The value of the current index }\left(\text { i.e. } i_{j}\right) \text { of } x \\
y_{\text {soln }} & - \text { The value of the current index }\left(\text { i.e. } i_{j}\right) \text { of } y \\
x_{\text {solnAddr }} & - \text { Contains the address of } x_{\text {soln }} \\
y_{\text {solnAddr }} & - \text { Contains the address of } y_{\text {soln }} \\
\text { AddrValue } & - \text { A buffer storing the address to be calculated/used }
\end{array}
$$

Moreover, we use following simple functions:

- $\operatorname{Addr}(s)$, where $s$ is one of the symbols $S, \$, C$, returns the unique address of the symbol $s$ on the input tape,

- Value $A t(a d d r)$, where $a d d r$ is an address, returns the symbol on the input tape at address $a d d r$,

- ReadIndex (index, addr), where index is a variable on the work tape and $a d d r$ is an address, copies the binary representation of an index $i_{j}$ which begins at address $a d d r$ into index; it also increments the address $a d d r$ such that it points to the first bit of $\left|i_{j+1}\right|$ if $j<n$ and to $\operatorname{Addr}(\$)$ if $j=n$.

Then Algorithm 1 will always halt with either a yes or a no because there is only a finite number of indexes encoded in $\alpha$ and hence in the case of notfinding a mismatch(including the mismatch due to one string finishing earlier than the other), the condition $a=b=\$$ will be satisfied giving a yes answer. The variables used in this algorithm are $x_{a d d r}, y_{a d d r}, x_{\text {soln }}, y_{\text {soln }}, x_{\text {soln Addr }}$, $y_{\text {solnAddr }}$ and AddrValue. All of them except for $x_{\text {soln }}$ and $y_{\text {soln }}$ are pointers to locations on read-tape and, hence, require only logarithmic space with respect to the input. We already know that $x_{\text {soln }}$ and $y_{\text {soln }}$ are within $\left\lceil\log _{2} k\right\rceil$ space and hence within logarithmic space with respect to the input. Since all the variables can be stored in space logarithmic with respect to the input, we conclude that $L_{1}$ can be decided in logarithmic space. We conclude that if $L_{1}$ is in LOG and $L_{2}$ is a singleton language, then $L_{1} \oplus L_{2}$ can be an undecidable language.

Theorem 3.5 can be extended to any time or space complexity class which contains LOG as well as to decidable languages. In particular, CS is not closed under directed extension of singleton languages.

Corollary 3.6. The family of context-sensitive languages is not closed under directed extension. More precisely, for $L_{x} \in C S$ the $L_{x}$-directed extension of a singleton language may not be decidable.

Corollary 3.7. The language classes NTIME, DTIME, NSPACE and DSPACE (all of which include LOG) are not closed under directed extension. More precisely, if $L_{x} \in N T I M E, D T I M E, N S P A C E$, DSPACE then the $L_{x}$-directed extension of a singleton language may not be decidable. 


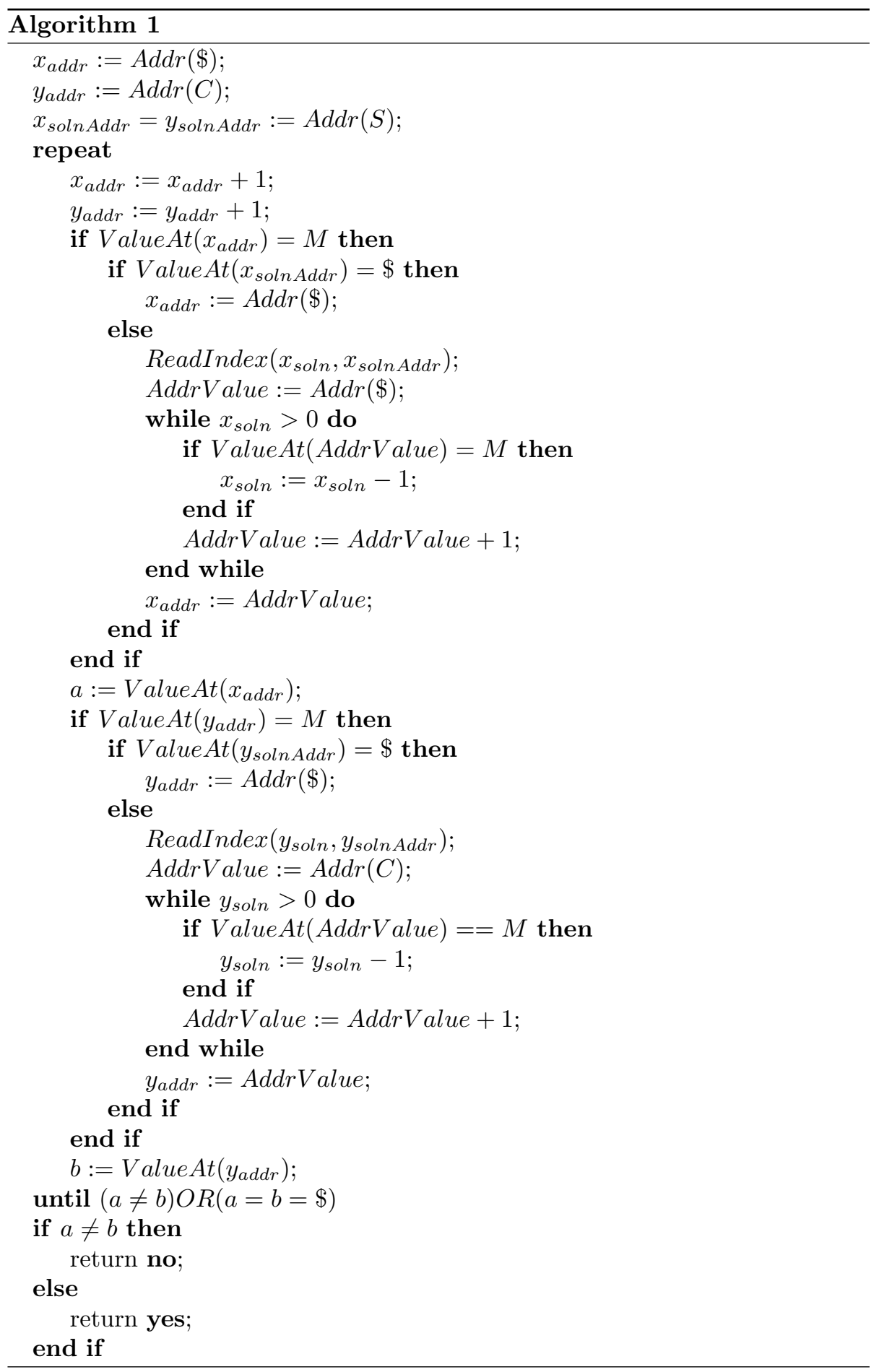


In Table 1 we summarize the results from this section. For two language classes $\mathcal{X}$ and $\mathcal{Y}$, it shows the language class $\mathcal{Z}$ from the Chomsky hierarchy such that for all $L_{x} \in \mathcal{X}$ and $L_{y} \in \mathcal{Y}$ we have $L_{x} \oplus L_{y} \in \mathcal{Z}$. Note that if we consider two language classes $\mathcal{X}, \mathcal{Y}$ which both contain the free monoid $\Sigma^{*}$ for any alphabet $\Sigma$, we will require that $\$ L \$=\$ L \$ \cap \$ \Sigma^{*} \$ \in \mathcal{Z}$ for all languages $L \in \mathcal{X}$ or $L \in \mathcal{Y}$ which are defined over $\Sigma$, due to Lemma 3.3. If we restrict ourselves to classes in the Chomsky hierarchy (or standard space/time complexity classes), this statement can be strengthend as $\mathcal{X} \cup \mathcal{Y} \subseteq \mathcal{Z}$. This shows that all entries in Table 2 can also be considered "lower bounds" for the language class $\mathcal{Z}$.

Finally, let us also note that if $L_{x}$ is a finite language, then $L_{x} \oplus L_{y}$ is finite for any $L_{y}$, even though it is not necessarily effectively finite if $L_{y}$ is undecidable.

\begin{tabular}{|c|c|c|c|c|}
\hline$L_{x} \backslash L_{y}$ & FIN or REG & $\mathrm{CF}$ & CS & $\mathrm{RE}$ \\
\hline REG & $\begin{array}{c}\text { REG } \\
(\text { Cor. } 3.2)\end{array}$ & $\begin{array}{c}\text { CF } \\
\text { (Cor. 3.2) }\end{array}$ & $\begin{array}{c}\text { CS } \\
\text { (Cor. 3.2) }\end{array}$ & $\begin{array}{c}\mathrm{RE} \\
\text { (Cor. 3.2) }\end{array}$ \\
\hline $\mathrm{CF}$ & $\begin{array}{c}\text { CF } \\
\text { (Cor. 3.2) }\end{array}$ & \multicolumn{2}{|c|}{$\begin{array}{c}\text { CS } \\
(\text { Thm. } 3.4)\end{array}$} & $\begin{array}{c}\mathrm{RE} \\
\text { (Cor. 3.2) }\end{array}$ \\
\hline CS & \multicolumn{4}{|c|}{$\begin{array}{c}\mathrm{RE} \\
\text { (Cor. 3.2 and Cor. 3.6) }\end{array}$} \\
\hline $\mathrm{RE}$ & \multicolumn{4}{|c|}{$\begin{array}{c}\mathrm{RE} \\
(\text { Cor. 3.2) }\end{array}$} \\
\hline
\end{tabular}

Table 1: Summary of closure properties: each entry shows which language class $L_{x} \oplus L_{y}$ belongs to if $L_{x}$ is from the corresponding language class in the left column and $L_{y}$ is from the corresponding language class in the top row.

\section{Equations and inverse operation}

In this section we investigate the following problem: Given two languages $L_{x}$, $L_{0}$ over $\Sigma^{*}$, does there exist a language $Y$ over $\Sigma^{*}$ such that $L_{x} \oplus Y=L_{0}$ ? Furthermore, we show how to effectively construct maximal and minimal solutions, with respect to the inclusion relation. Throughout this section, we consider the languages $L_{x}$ and $L_{0}$ to be constants. For the equation $L_{x} \oplus Y=L_{0}$ we call a language $L_{y}$ a solution if it satisfies $L_{x} \oplus L_{y}=L_{0}$.

We can use the canonical right-inverse of the directed extension in order to decide the existence of a solution as well as to find the maximal solution. The canonical right-inverse of an arbitrary binary language operation " + " is the binary language operation " $"$ " defined as

$$
x-w=\left\{y \in \Sigma^{*} \mid w \in x+y\right\} .
$$

It was proved that proved that, if there exists a solution $L_{y}$ of the equation $L_{x}+Y=L_{0}$, then $L_{\max }=\left(L_{x}-L_{0}^{c}\right)^{c}$ is also a solution, and every other solution $L_{y}^{\prime}$ of this equation is contained in $L_{\max }$ [13]. In other words, for 
languages $L_{x}, L_{y}$, and $L_{0}$

$$
L_{x}+L_{y}=L_{0} \Longleftrightarrow L_{y} \subseteq\left(L_{x}-L_{0}^{c}\right)^{c} .
$$

It is easy to see that the right-inverse of directed extension is

$$
\begin{aligned}
x \ominus w & =\left\{y \in \Sigma^{*} \mid w \in x \oplus y\right\} \\
& = \begin{cases}\operatorname{Pref}(w) & \text { if } x=\alpha w \\
\emptyset & \text { otherwise. }\end{cases}
\end{aligned}
$$

Therefore, we obtain that $L_{\max }=\left(L_{x} \ominus L_{0}^{c}\right)^{c}$ is the maximal solution (with respect to inclusion) of ( $\left.L_{x} \oplus Y=L_{0}\right)$ if and only if $L_{x} \oplus Y=L_{0}$ has at least one solution.

This already implies that we can decide whether or not the equation $L_{x} \oplus Y=$ $L_{0}$ has a solution $L_{y}$. Yet, we want to present a "more direct" approach to test solvability of this equation: we will show that the equation has a solution if and only if $L_{x} \oplus L_{0}=L_{0}$.

Theorem 4.1. The equation $L_{x} \oplus Y=L_{0}$ has a solution $L_{y}$ if and only if $L_{0}$ is a solution as well.

Proof. Trivially, if $L_{x} \oplus L_{0}=L_{0}$, then there exists an $L_{y}$ such that $L_{x} \oplus L_{y}=L_{0}$.

Conversely, we need to prove that if $L_{x} \oplus L_{y}=L_{0}$, then $L_{x} \oplus L_{0}=L_{x} \oplus L_{y}$. Let us consider a string $w \in L_{x} \oplus L_{y}$. This implies that $w$ is a suffix of a word $x \in L_{x}$ and, therefore, $w \in x \oplus w \subseteq L_{x} \oplus L_{0}$. This proves that $L_{x} \oplus L_{0} \supseteq L_{x} \oplus L_{y}$.

Now, take any $w^{\prime} \in L_{x} \oplus w$ for some $w \in L_{0}=L_{x} \oplus L_{y}$. Hence, $w^{\prime}$ is a suffix of some word $x \in L_{x}$ and, furthermore, there exists a word $y \in L_{y}$ which is a prefix of $w$ which in turn is a prefix of $w^{\prime}$ by Lemma 3.1. Clearly, this implies that $w^{\prime} \in x \oplus y \subseteq L_{x} \oplus L_{y}$. We conclude $L_{x} \oplus L_{0}=L_{x} \oplus L_{y}$.

Next, we investigate solutions which are minimal with respect to inclusion; that is, a solution $L_{y}$ of the equation $L_{x} \oplus Y=L_{0}$ is minimal if for all words $y \in L_{y}$ the language $L_{y} \backslash\{y\}$ is not a solution: $L_{x} \oplus\left(L_{y} \backslash\{y\}\right) \neq L_{0}$. We present a general method to find a minimal solution if we already know one solution.

Theorem 4.2. If $L_{x} \oplus Y=L_{0}$ has the solution $L_{y}$, then $L_{\min }=\left(L_{y} \backslash L_{y} \Sigma^{+}\right) \cap$ $\operatorname{Inf}\left(L_{x}\right)$ is a minimal solution.

Proof. First, let us show that $L_{\min }$ is indeed a solution. Because $L_{\min } \subseteq L_{y}$, we have $L_{x} \oplus L_{\text {min }} \subseteq L_{x} \oplus L_{y}=L_{0}$. Vice versa, for every $w \in L_{0}$ there exists $x \in L_{x}$ and $y \in L_{y}$ such that $w \in x \oplus y$. Let $y^{\prime}$ be the shortest prefix of $y$ such that $y^{\prime} \in L_{y}$. Because $y^{\prime}$ does not have a shorter prefix in $L_{y}$ and because $y^{\prime}$ is an infix of $x$, we obtain that $y^{\prime} \in L_{\text {min }}$. Now, since $y^{\prime}$ is also a prefix of $w$, we obtain that $w \in x \oplus y^{\prime} \subseteq L_{x} \oplus L_{\text {min }}$.

For the sake of obtaining a contradiction, let us assume that $L_{\min }$ is not a minimal solution. This implies that either (a) there is $y \in L_{\min }$ such that $L_{x} \oplus y=\emptyset$ or (b) there are two distinct strings $y_{1}, y_{2} \in L_{\text {min }}$ such that a word $w$ in $L_{x} \oplus y_{1} \cap L_{x} \oplus y_{2}$ exists. Case (a) does not hold because it would imply 
that $y$ is not an infix of any word in $L_{x}$. Case (b) implies that $y_{1}$ and $y_{2}$ are both prefixes of the word $w$ which means that we may assume that $y_{1}$ is a prefix of $y_{2}$ without loss of generality. Since both words have to belong to $L_{y}$ and $y_{2} \in y_{1} \Sigma^{*}$, we conclude that $y_{2} \notin L_{m i n}$ - a contradiction.

From the two results in this section, Theorems 4.1 and 4.2 , we infer that if the equation $L_{x} \oplus Y=L_{0}$ has a solution, then $L_{0, \min }=\left(L_{0} \backslash L_{0} \Sigma^{+}\right) \cap \operatorname{Inf}\left(L_{x}\right)$ is a minimal solution.

\section{Discussion and conclusions}

We now compare the directed extension operation with two other formal language operations that are biologically motivated and extend strings: the PA-matching operation and the superposition operation. The PA-matching operation is a binary operation proposed by Kobayashi et al [20] and inspired by the PA-match operation that was part of Parallel Associate Memory(PAM) model proposed by Reif [32]. The PA-matching operation is meant to be implemented by some recombinant DNA processes and is defined as follows. Given two words $x \in V_{1}^{+}$and $y \in V_{2}^{+}$, the result of the PA-matching between $x$ and $y$ is defined as:

$P A m(x, y)=\left\{u v \mid x=u w, y=w v\right.$, for some $w \in\left(V_{1} \cap V_{2}\right)^{+}$, and $\left.u \in V_{1}^{*}, v \in V_{2}^{*}\right\}$

Note that PA-matching results in the extension of a the word $x$ by a suffix of $y$, if $x$ has a suffix which is the same with a prefix of $y$. The main difference between this operation and directed extension is that here the common suffix/prefix that guides the extension is deleted from the result, while in the case of directed extension no deletion takes place.

The superposition operation is a binary operation proposed by Bottoni et al in [3] and can be implemented by the use of the DNA Polymerase enzyme. The result of the superposition operation between words $x \in V_{1}^{+}$and $y \in V_{2}^{+}$, denoted by $x \diamond y$, consists of the set of all words $z \in\left(V_{1} \cup \bar{V}_{2}\right)^{+}$defined as follows ( $\bar{y}$ denotes the complement of $y$, that is, the image of $y$ through a morphic involution):

1. If there exist $u \in V_{1}^{*}, w \in V_{1}^{+}, v \in V_{2}^{*}$ such that $x=u w, y=\bar{w} v$, then $z=u w \bar{v}$.

2. If there exist $u, v \in V_{1}^{*}$ such that $x=u \bar{y} v$, then $z=u \bar{y} v$.

3. If there exist $u \in V_{2}^{*}, w \in V_{1}^{*}$ such that $x=w v, y=u \bar{w}$, then $z=\bar{u} w v$.

4. If there exist $u, v \in V_{2}^{*}$ such that $y=u \bar{x} v$, then $z=\bar{u} x \bar{v}$.

The superposition operation also extends words but, in the case of superposition the extension can be bidirectional, while in the case of directed extension the extension is always uni-directional. This and other differences lead to the 
two operations being different, as illustrated by the difference in the closure properties of the two operations.

Table 2 summarizes the closure properties of the operations of directed extension, PA-matching and superposition.

\begin{tabular}{|l|c|c|c|}
\hline Class of $L_{x}$ and $L_{y}$ & $\oplus$ & $P A m$ & $\diamond$ \\
\hline Regular & Closed & Closed & Closed \\
\hline Context Free & Not Closed & Not Closed & Not Closed \\
\hline Context Sensitive & Not Closed & Not Closed & Closed \\
\hline Recursively Enumerable & Closed & Closed & Closed \\
\hline
\end{tabular}

Table 2: Closure properties under the directed extension operation, $\oplus$, compared to the PA-Matching and superposition operations.

We end this paper by several remarks on iterated directed extension. When investigating language operations, it is common to investigate an iterated version of the operation as well. In particular, when studying biologically motivated operations as is the case here, the iterated version is sometimes the operation that better reflects the biological phenomenon in question (DNA replication) or experimental lab protocols (Polymerase Chain Reaction). Let us present here three natural versions of the iterated directed extension. We define

1. the iterated self-directed extension of $L$ as $\mu^{*}(L)=\lim _{n \rightarrow \infty} \mu^{n}(L)$ where $\mu(L)=L \cup(L \oplus L)$,

2. the $L$-iteration-directed extension of $L_{y}$ as $\nu_{L_{y}}^{*}(L)=\lim _{n \rightarrow \infty} \nu_{L_{y}}^{n}(L)$ where $\nu_{L_{y}}(L)=L \cup\left(L \oplus L_{y}\right)$, and

3. the iterated $L_{x}$-directed extension of $L$ as $\xi_{L_{x}}^{*}(L)=\lim _{n \rightarrow \infty} \xi_{L_{x}}^{n}(L)$ where $\xi_{L_{x}}(L)=L \cup\left(L_{x} \oplus L\right)$.

Here, we use the notation that for any domain $D$ and function $h: D \rightarrow D$ we have $h^{0}(L)=L$ and $h^{i}(L)=h\left(h^{i-1}(L)\right)$ for $i \geq 1$.

Let us show that in all three cases we have $h^{*}(L)=h(L)$ for $h \in\left\{\mu, \nu_{L_{y}}, \xi_{L_{x}}\right\}$ which means that the results that we obtained in this paper can easily be extended to the iterated versions. Indeed, the only difference is that we add the term $h^{0}(L)=L$ to the directed extension.

For case 1.) consider a word $w \in \mu^{2}(L)$, that is (a) $w \in \mu(L)$ or (b) $w=x \oplus y$ for $x, y=\mu(L)=L \cup(L \oplus L)$. If (b) holds, we obtain from Lemma 3.1 that there exists $x^{\prime} \in L$ such that $x$ is a suffix of $x^{\prime}$ an $y^{\prime} \in L$ such that $y^{\prime}$ is a prefix of $y$ (note that we do allow $x=x^{\prime}$ or $y=y^{\prime}$ ). Clearly, we also have $w \in x^{\prime} \oplus y^{\prime}$ and may conclude that $w \in L \oplus L \subseteq \mu(L)$. This implies that $\mu^{2}(L) \subseteq \mu(L)$ and, due to the inductive definition of $\mu^{i}$ we have $\mu^{i}(L)=\mu(L)$ for any $i \geq 1$. We conclude that $\mu^{*}(L)=\mu(L)$. The result follows by analogous arguments for the cases 2.) and 3.). 


\section{References}

[1] A. Alhazov, A. Krassovitskiy, Y. Rogozhin, and S. Verlan. P systems with insertion and deletion exo-operations. Fundamenta Informaticae, 110(14):13-28, 2011.

[2] A. Alhazov, A. Krassovitskiy, Y. Rogozhin, and S. Verlan. P systems with minimal insertion and deletion. Theoretical Computuer Science, 412(12):136-144, 2011.

[3] P. Bottoni, A. Labella, V. Manca, and V. Mitrana. Superposition based on Watson-Crick-like complementarity. Theory of Computing Systems, 39(4):503-524, 2006.

[4] D. Cheptea, C. Martín-Vide, and V. Mitrana. A new operation on words suggested by DNA biochemistry: hairpin completion. In Transgressive Computing, TC 2006, pages 216-228, 2006.

[5] M. Daley, L. Kari, G. Gloor, and R. Siromoney. Circular contextual insertions/deletions with applications to biomolecular computation. In Proceedings of 6th International Symposium on String Processing and Information Retrieval, SPIRE 1999, pages 47-54, 1999.

[6] R. Freund, M. Kogler, Y. Rogozhin, and S. Verlan. Graph-controlled insertion-deletion systems. In I. McQuillan and G. Pighizzini, editors, Proceedings of 12th International Workshop on Descriptional Complexity of Formal Systems, DCFS 2010, volume 31 of Electronic Proceedings in Theoretical Computer Science, pages 88-98, 2010.

[7] R. Freund, Y. Rogozhin, and S. Verlan. P systems with minimal left and right insertion and deletion. In J. Durand-Lose and N. Jonoska, editors, Proceedings of 11th International Conference on Unconventional Computation and Natural Computation, UCNC 2012, volume 7445 of LNCS, pages 82-93, 2012.

[8] R. Freund, Y. Rogozhin, and S. Verlan. Generating and accepting P systems with minimal left and right insertion and deletion. Natural Computing, 13(2):257-268, 2014.

[9] R. W. Gatterdam. Splicing systems and regularity. International Journal of Computer Mathematics, 31(1-2):63-67, 1989.

[10] T. Head. Formal language theory and DNA: an analysis of the generative capacity of specific recombinant behaviors. Bulletin of Mathematical Biology, 49(6):737-759, 1987.

[11] T. Head, D. Pixton, and E. Goode. Splicing systems: regularity and below. In M. Hagiya and A. Ohuchi, editors, Revised Papers from the 8th International Workshop on DNA Based Computers: DNA Computing, DNA 8, volume 2568 of $L N C S$, pages $262-268,2003$. 
[12] S. Hussini, L. Kari, and S. Konstantinidis. Coding properties of DNA languages. Theoretical Computer Science, 290(3):1557-1579, 2003.

[13] L. Kari. On language equations with invertible operations. Theoretical Computer Science, 132(1-2):129-150, 1994.

[14] L. Kari, R. Kitto, and G. Thierrin. Codes, involutions, and DNA encodings. In W. Brauer, H. Ehrig, J. Karhumaki, and A. Salomaa, editors, Formal and Natural Computing, volume 2300 of LNCS, pages 376-393. 2002.

[15] L. Kari and S. Kopecki. Deciding whether a regular language is generated by a splicing system. In D. Stefanovic and A. Turberfield, editors, DNA Computing and Molecular Programming, volume 7433 of LNCS, pages 98109. 2012.

[16] L. Kari and E. Losseva. Block substitutions and their properties. Fundamenta Informaticae, 73(1-2):165-178, 2006.

[17] L. Kari, G. Păun, G. Thierrin, and S. Yu. At the crossroads of DNA computing and formal languages: characterizing recursively enumerable languages using insertion-deletion systems. In DNA Based Computers III (DNA3), volume 48 of DIMACS: Series in Discrete Mathematics and Theoretical Computer Science, pages 329-347. 1999.

[18] L. Kari and P. Sosík. On the weight of universal insertion grammars. Theoretical Computer Science, 396(1-3):264-270, 2008.

[19] S. Kim. An algorithm for identifying spliced languages. In D. Lee and S.H. Teng, editors, Proceedings of 11th Annual International Symposium on Algorithms and Computation, ISAAC 2000, volume 1276 of LNCS, pages 403-411, 1997.

[20] S. Kobayashi, V. Mitrana, G. Păun, and G. Rozenberg. Formal properties of PA-matching. Theoretical Computer Science, 262(1-2):117-131, 2001.

[21] S. Kopecki. On iterated hairpin completion. Theoretical Computer Science, 412(29):3629-3638, 2011.

[22] A. Krassovitskiy, Y. Rogozhin, and S. Verlan. Computational power of P systems with small size insertion and deletion rules. In T. Neary, D. Woods, A. K. Seda, and N. Murphy, editors, Proceedings of International Workshop on The Complexity of Simple Programs, CSP 2008, volume 1 of Electronic Proceedings in Theoretical Computer Science, pages 108-117, 2008.

[23] A. Krassovitskiy, Y. Rogozhin, and S. Verlan. Further results on insertiondeletion systems with one-sided contexts. In C. Martín-Vide, F. Otto, and H. Fernau, editors, Proceedings of 2nd International Conference on Language and Automata Theory and Applications, LATA 2008, volume 5196 of LNCS, pages 333-344, 2008. 
[24] A. Krassovitskiy, Y. Rogozhin, and S. Verlan. Computational power of insertion-deletion (P) systems with rules of size two. Natural Computing, 10(2):835-852, 2011.

[25] F. Manea, C. Martín-Vide, and V. Mitrana. On some algorithmic problems regarding the hairpin completion. Discrete Applied Mathematics, 157(9):2143-2152, 2009.

[26] F. Manea and V. Mitrana. Hairpin completion versus hairpin reduction. In S. B. Cooper, B. Löwe, and A. Sorbi, editors, Proceedings of 3rd Conference on Computability in Europe, CiE 2007, volume 4497 of LNCS, pages 532541, 2007.

[27] M. Margenstern, G. Păun, Y. Rogozhin, and S. Verlan. Context-free insertion-deletion systems. Theoretical Computer Science, 330(2):339-348, 2005.

[28] A. Matveevici, Y. Rogozhin, and S. Verlan. Insertion-deletion systems with one-sided contexts. In J. Durand-Lose and M. Margenstern, editors, Proceedings of 5th International Conference on Machines, Computations, and Universality, MCU 2007, volume 4664 of LNCS, pages 205-217. 2007.

[29] D. Pixton. Regularity of splicing languages. Discrete Applied Mathematics, 69(1-2):101-124, 1996.

[30] G. Păun, M. J. Pèrez-Jimènez, and T. Yokomori. Representations and characterizations of languages in Chomsky hierarchy by means of insertiondeletion systems. International Journal of Foundations of Computer Science, 19(4):859-871, 2008.

[31] G. Păun, G. Rozenberg, and A. Salomaa. DNA Computing: New Computing Paradigms(Texts in Theoretical Computer Science. An EATCS Series). Springer-Verlag New York, Inc., 2006.

[32] J. H. Reif. Parallel molecular computation. In Proceedings of the 7th Annual ACM Symposium on Parallel Algorithms and Architectures, SPAA 1995, pages 213-223, 1995.

[33] A. Takahara and T. Yokomori. On the computational power of insertiondeletion systems. Natural Computing, 2(4):321-336, 2003.

[34] M. Yong, J. Xiao-Gang, S. Xian-Chuang, and P. Bo. Minimizing of the only-insertion insdel systems. Journal of Zhejiang University Science A, 6(10):1021-1025, 2005. 